\title{
CORRECTION
}

\section{Correction: Photon upconversion: from two-photon absorption (TPA) to triplet-triplet annihilation (TTA)}

Cite this: Phys. Chem. Chem. Phys. 2016, 18, 7537

Changqing Ye, Liwei Zhou, Xiaomei Wang* and Zuoqin Liang

DOI: $10.1039 / \mathrm{c} 6 \mathrm{cp} 90051 \mathrm{~h}$

Correction for 'Photon upconversion: from two-photon absorption (TPA) to triplet-triplet annihilation

www.rsc.org/pccp

(TTA)' by Changqing Ye et al., Phys. Chem. Chem. Phys., 2016, DOI: 10.1039/c5cp07296d.

Fig. 1 in the published article should be replaced with the one shown below:

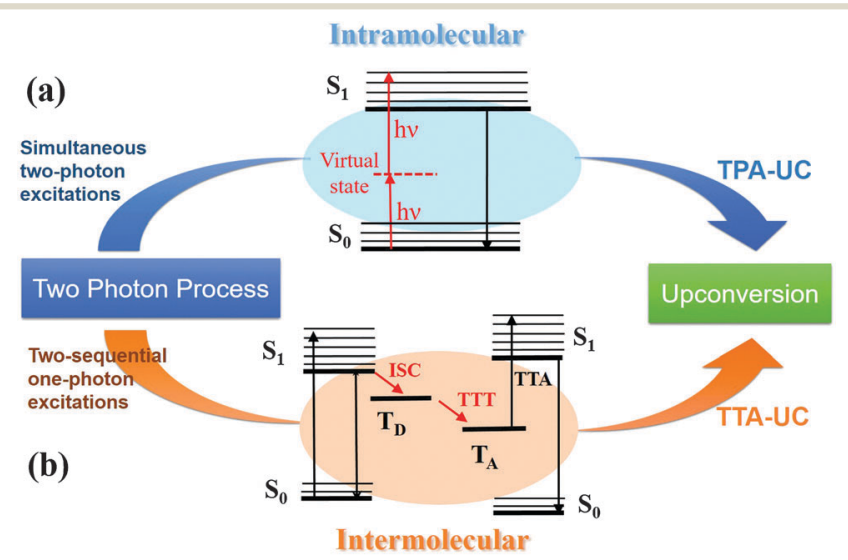

Fig. 1 Jablonski diagrams of TPA-UC (dual energy level model) (a) and TTA-UC (b).

The Royal Society of Chemistry apologises for these errors and any consequent inconvenience to authors and readers. 